\title{
On-line Signature Verification Based on Modified Dynamic Time Warping and Wavelet Sub-band Coding
}

\author{
Juan Carlos Sánchez-Diaz ${ }^{1}$, Juan Manuel Ramírez-Cortes ${ }^{1}$, \\ Rogerio Enriquez-Caldera ${ }^{1}$, and Pilar Gomez-Gil ${ }^{2}$ \\ ${ }^{1}$ Department of Electronics; National Institute of Astrophysics, Optics and Electronics. \\ Luis Enrique Erro No. 1 Tonantzintla, Puebla. 72840. Mexico \\ ${ }^{2}$ Department of Computer Science, National Institute of Astrophysics, \\ Optics and Electronics. Luis Enrique Erro No. 1 Tonantzintla, Puebla. 72840. Mexico \\ karnaught@hotmail.com, jmram@inaoep.mx, rogerio@inaoep.mx, \\ pgomez@inaoep.mx
}

\begin{abstract}
This paper presents an on-line signature biometric system based on a modified Dynamic Time Warping (DTW) algorithm applied to the signature wavelet coefficients. The modification on DTW relies on the use of direct matching points information (DMP) to dynamically adapt the similarity measure during the matching process, which is shown to increase the verification success rate. The wavelet analysis is done using a sub-band coding algorithm at global and local level. The use of wavelet coefficients showed a considerable reduction in processing time and an improvement in the equal error recognition rate (EER). The system was tested using a locally constructed database. A comparison of the ROC curves obtained in each case is presented.
\end{abstract}

Keywords: Signature, online, verification, dynamic time warping, wavelet.

\section{Introduction}

Automatic personal identification and verification based on biometrics has received extensive attention in past years. Biometric identification refers to identifying an individual based on physiological or behavioral characteristics. It associates/disassociates an individual with a previously determined identity based on how one is or what one does. Identification can be in the form of verification, which entails authenticating a claimed identity, or recognition, which entails determining the identity of a given person from a database of persons known to the system [1].

A biometric system aims to provide automatic recognition of an individual based on features or characteristics unique to each human being. Biometric systems are based on several modalities, such as iris, face, ear shape, hand-shape, fingerprints, palm prints [2-5], or dynamical features like gait, on-line signature verification [6-7], or combination of them [8]. Requirements, strengths, and weaknesses of each modality have been widely reported in the literature.

Among the different existing forms of biometrics, signature-based verification has the advantage that signature analysis requires no invasive measurements and it is 
widely accepted since signature has long been established as the most popular mean for personal verification in a variety of contexts, including commerce applications, banking transactions, legalization of contracts, and others. Signature is a behavioral biometric, which means that it is not based on physical properties of the individual, such as face, hand-shape, or fingerprint. A signature may change over time and it is not as unique or difficult to forge as iris patterns or fingerprints, however, acceptance by the public makes it more suitable for certain lower-security authentication needs. Moreover, PDA and other portable digital devices are capable of providing support to get information about specific characteristics from signatures

Signature verification is split into two categories according to the available input data. Offline signature verification takes as input the image of a signature and is useful in automatic verification of signatures found on bank checks and documents. Online signature verification uses signatures that are captured by pressure-sensitive tablets that extract dynamic properties of a signature in addition to its shape [9]. The signature can be regarded as a series of rapid movements, which are dependent on the properties of human neuromuscular system. Mapping of the personal hand cadence and movement during the writing is highly difficult to forge. Dynamic features include the number and order of the strokes, the overall speed of the signature, the pen pressure at each point, cadence, etc., and make the signature more unique and more difficult to forge. As a result, online signature verification could be more reliable than offline signature verification in most cases. Various approaches have been proposed to solve the online signature verification problem: Multilayer perceptron neural networks [10], Hidden Markov Models [11], neurofuzzy systems [12], wavelet transform followed by discrete cosine transform for dimensionality reduction [13], fusion of methods, such as dynamic time warping and Hidden Markov Models [14], or dynamic time warping improved by incorporating the use of Fourier descriptors [15].

In this work, an on line signature verification with a feature extraction based on discrete wavelet transform is presented. The matching is performed by a modified dynamic time warping algorithm (MDTW), which operates on the approximation coefficients obtained through a wavelet sub-band coding algorithm. Two type of signature analysis are allowed by applying the matching at the stroke level (local analysis) or using the whole signature at once (global analysis).

\section{System Description}

System description can be summarized using the block diagram of Figure 1. In order to minimize the fluctuations of place, size, and rotation of the signature, some preprocessing operations were included in the first block. The rotation normalization was implemented using the Hotelling transform, which performs an alignment of the signature with its main axis through a matrix transformation formed by the eigenvectors of the covariance matrix using the $\mathrm{X}-\mathrm{Y}$ signature position data. Once this tasks are performed, magnitude and phase information from the normalized data are used as input function to the recognition system. If a stroke-based analysis is performed, signature splitting is carried out in this block using the pen-up feature of the digitizing tablet. In the next block, feature extraction is performed using time-scale decomposition up to the specified level, based on the wavelet sub-band coding algorithm. A 
modified dynamic time warping (MDTW) algorithm is then applied on the wavelet coefficients. This operation consists of a matching/warping operation between vectors, which simultaneously finds a dissimilitude value between them. In the last stage, a matching decision block takes the dissimilitude value from the feature extraction blocks and compares it against a threshold value which is dynamically calculated based on statistical data information. If the dissimilitude value obtained from MDTW is less than threshold value, signature matching is decided to be positive. The template block temporarily stores the template signature, statistical data information, and dissimilitude values found in the training stage, for their posterior use during the verification process.

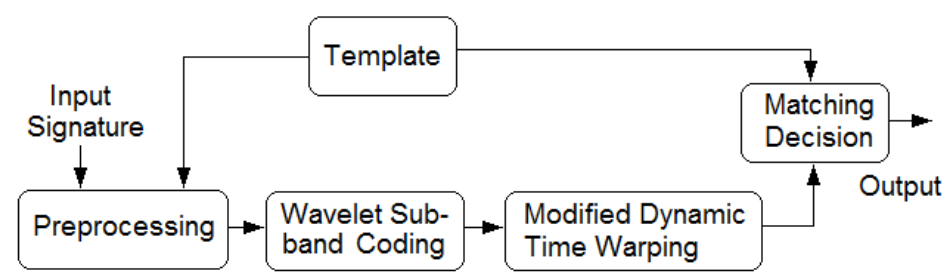

Fig. 1. System block diagram

\section{Wavelet Sub-band Coding}

The Discrete Wavelet Transform (DWT) is used to analyze the temporal and spectral properties of non-stationary signals. The DWT is defined by the following equation [16]:

$$
W(j, k)=\sum_{j} \sum_{k} f(x) 2^{-j / 2} \psi\left(2^{-j} x-k\right)
$$

The set of functions $\psi_{j, k}(n)$ is referred to as the family of wavelets derived from $\psi(n)$, which is a time function with finite energy and fast decay called the mother wavelet. The basis of the wavelet space corresponds then, to the orthonormal functions obtained from the mother wavelet after scale and translation operations. The definition indicates the projection of the input signal into the wavelet space through the inner product, then, any function $f(x) \in \mathrm{L}^{2}(R)$ can be represented in the form:

$$
f(x)=\sum_{j, k} d_{j}(k) \psi_{j, k},
$$

where $d_{j}(k)$ are the wavelet coefficients at level $\mathrm{j}$. The coefficients at different levels can be obtained through the projection of the signal into the wavelets family as:

$$
\begin{gathered}
\left\langle f, \psi_{j, k}\right\rangle=\sum_{l} d_{l}\left\langle f, \phi_{j, k+l}\right\rangle \\
\left\langle f, \phi_{j, k}\right\rangle=\frac{1}{\sqrt{2}} \sum_{l} c_{l}\left\langle f, \phi_{j-1,2 k+l}\right\rangle
\end{gathered}
$$


The DWT analysis can be performed using a fast, pyramidal algorithm described in terms of multirate filter banks [17]. The DWT can be viewed as a filter bank with octave spacing between filters. Each sub-band contains half the samples of the neighboring higher frequency sub-band. In the pyramidal algorithm the signal is analyzed at different frequency bands with different resolution by decomposing the signal into a coarse approximation and detail information. The coarse approximation is then further decomposed using the same wavelet decomposition step. This is achieved by successive high-pass and low-pass filtering of the time signal, and a down-sampling by two as defined by the following equations:

$$
\begin{aligned}
& c_{j}(k)=\sum_{m} h(m-2 k) c_{j+1}(m) \\
& d_{j}(k)=\sum_{m} g(m-2 k) c_{j+1}(m)
\end{aligned}
$$

Figure 2 shows a one-level filter bank. Signals $c_{j}(k)$, and $d_{j}(k)$ are known as approximation and detail coefficients, respectively. This process may be executed iteratively forming a wavelet decomposition tree up to any desired resolution level.

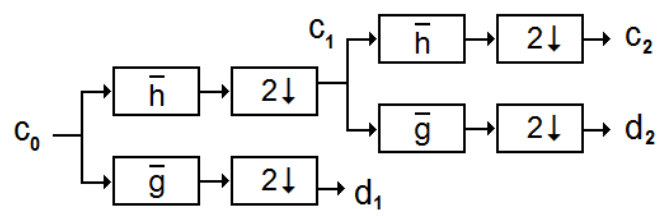

Fig. 2. Two-level discrete wavelet filter bank scheme

In this work, the approximation coefficients were used as input to the modified dynamic time warping algorithm, which is described in the following section. Different experiments were made using several wavelets and decomposition levels to obtain the best system performance, as described in the results section.

\section{Modified Dynamic Time Warping Algorithm}

Dynamic Time Warping was introduced by Kruskal and Liberman in the context of speech recognition [18], as a computational technique to make a matching between two time series, which may have different number of samples, providing a normalization and alignment of both sequences. DTW can distort the time axis by compressing it at some places and expanding it at others, as required. The main objective is the optimization of a function cost used to travel from one point to another, giving an optimal matching path based on some constraints. Minimization of the function cost is described as:

$$
D\left(T_{x}, T_{y}\right)=\min _{\phi_{x}, \phi_{y}} \sum_{k=1}^{T} d\left(\phi_{x}(k), \phi_{y}(k)\right) m(k)
$$


Where $d\left(\phi_{x}(k), \phi_{y}(k)\right)$ is a dissimilitude value between both time sequences in the warping trajectory $\mathrm{T}$, and $m(k)$ is a local weighting factor. Normalization of this measure is done by dividing this value between a global weight factor, which corresponds to the number of points in the warping trajectory, as expressed in equation 8 . Details can be checked in reference [18].

$$
D_{v}=\frac{D\left(T_{x}, T_{y}\right)}{T}
$$

In this work we propose some modifications to the classical algorithm. The first one is the incorporation of a warping diagonal deviation used as complementary normalization factor. This value is given by equation 9 , and expresses the ratio of the number of points in the warping trajectory and the sum of the number of points on the original data vectors to be matched. As the two data sequences are more dissimilar, the warping path separates from the diagonal and the coefficient tends to one.

$$
D_{N}=\frac{T}{T_{x}+T_{y}}
$$

This dissimilitude measurement was also enhanced using information about direct matching points (DMP). DMPs are matched points unambiguously defined between data sets. Figure 3 shows an example of a matching segment between two data sequences, with the DMPs plotted in solid lines.

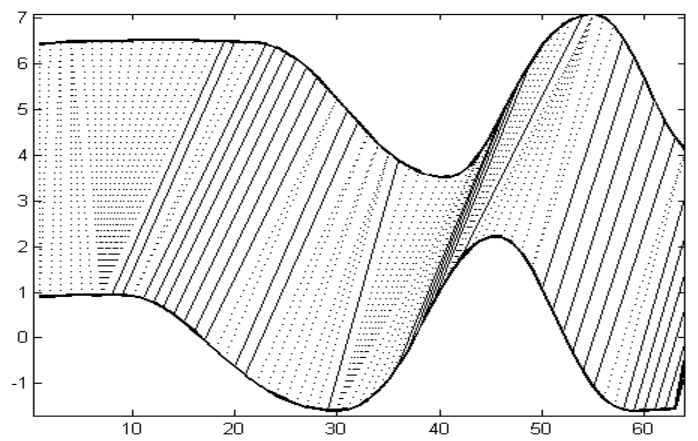

Fig. 3. Example of DMPs between two trajectories

Using these particular points, we propose an additional matching coefficient defined as:

$$
C_{d m p}=1-\frac{\sum D M P}{T},
$$

where DMP stands for direct matching points, and $\mathrm{T}$ is the number of points in the warping trajectory. This coefficient is used as an additional weight factor in the dissimilitude measurement of DTW. The dissimilitude value $V_{M D T W}$ obtained through the modified dynamic time warping is finally defined as: 


$$
V_{M D T W}=\left(D_{v}\right)\left(D_{N}\right)\left(C_{d m p}\right)=\frac{\left(D\left(T_{x}, T_{y}\right)\right)\left(1-\sum d m p\right)}{T\left(T_{x}+T_{y}\right)}
$$

This value is used to make a decision on whether the signature corresponds to the template defined by the user in each case, by comparing it to a threshold value $\alpha_{t h}$ defined in equation 12 .

$$
\alpha_{t h}=\overline{T m}+K \sigma_{T m},
$$

where $\overline{T m}$ is template mean value, $\sigma_{T m}$ is the template standard deviation value, and $K$ is a weight factor used to control the trade-off between false rejections and false acceptances. $K$ is defined by the administrator of the biometric system according to the need for security, which depends on some risk evaluation. During the system evaluation process this parameter assumes the range which allows the system to give both, FAR and FRR values from 0 to $100 \%$.

$$
\text { matching }=\left\{\begin{array}{l}
\text { positive if } V_{M D T W} \leq \alpha_{t h} \\
\text { negative if } V_{M D T W}>\alpha_{t h}
\end{array}\right.
$$

Figure 4 show an example of warping trajectory (white line) on a dissimilitude matrix with values represented in gray levels.

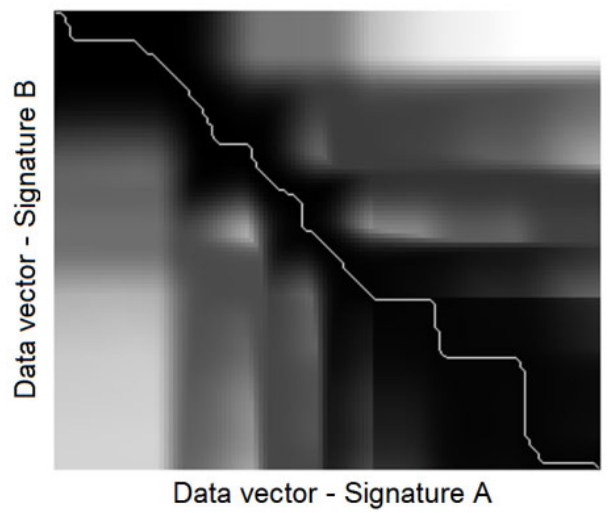

Fig. 4. Warping trajectory on a dissimilitude matrix obtained from two data vectors

\section{Experimental Setup}

On-line signature data acquisition was accomplished using a digitizing tablet Genius G-PEN 340 with a 3 X4 inches active area and maximum data transmission rate of 100 points per second. For the described experiments a local signature database consisting of 1000 genuine signatures from 50 signers and 240 skilled forgeries from 12 subjects, was generated. The biometric system is accessed through a graphical user 
interface developed in MATLAB. Figure 5 shows the main screen of the application. Two modalities can be used: The first one consists of the signature analysis at strokelevel or using the whole signature. The second one is the application of the proposed modified dynamic time working algorithm directly to the raw data, or to the wavelet coefficients. From the combination of these modalities, four type of analysis can be performed. The graphical user interface allows the following tasks: Organize signature data files, set the number of signers and signatures to be used, generate templates, set the analysis type, perform a global analysis between enrolled signatures, perform signature verification and perform signature recognition. Figure 5 shows the GUI window in the verification mode:

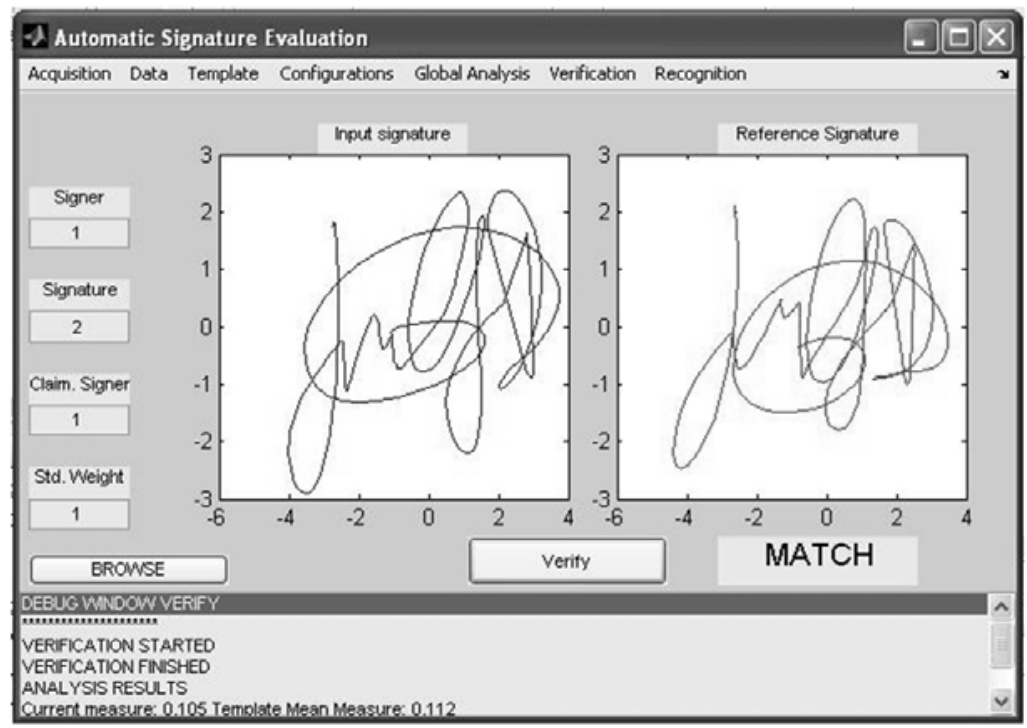

Fig. 5. Main screen of the graphical user interface in verification mode

\section{Results}

Evaluation of the system performance was done through the ROC plot (receiver operation characteristic) for several cases. ROC is a plot of the false acceptance ratio (FAR) versus the false rejection ratio (FFR) [19]. The equal error rate (EER) is obtained from the point in which FAR and FFR assume the same value. Figure 6 shows the ROC curve obtained from an experiment which was done to check the effect of using the modified dynamic time warping algorithm vs. the classical DTW. The test was done using the raw data corresponding to the whole signature, i.e., without using wavelet decomposition. From this plot it can be seen that MDTW showed an EER of $12.28 \%$ approximately. 


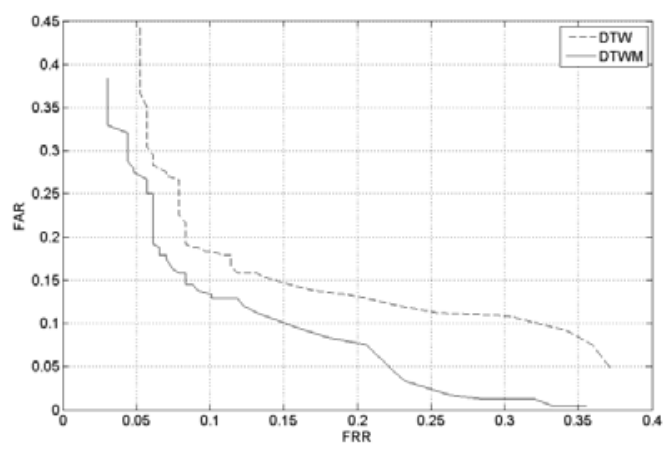

Fig. 6. Classical DTW vs Modified DTW ROC curves

A second experiment was done in order to characterize the system performance when the wavelet decomposition was incorporated. Figure 7 shows the ROC plot obtained using a different wavelet mother in each case, with a 3-level wavelet decomposition. This figure shows that the best result was obtained using the Coifflet- 3 wavelet.

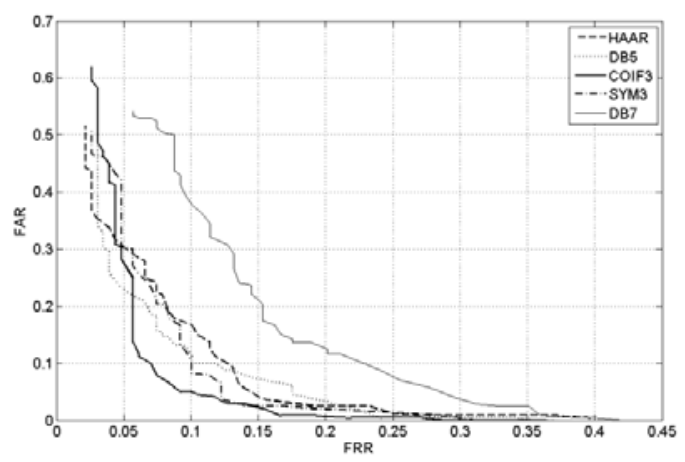

Fig. 7. ROC curves obtained using different wavelets

Figure 8 shows the improvement in performance obtained when the level-3 wavelet decomposition is incorporated into the biometric system. The plot shows an $\mathrm{EER}=7.46 \%$ for that case. In both cases, the proposed modified dynamic time warping algorithm is applied. The wavelet decomposition helped also to obtain a reduction in the dimensionality of data, which impacted on getting an improvement in the execution time.

Finally, figure 9 shows a comparison of ROC curves obtained when the system is tested using the stroke-based analysis. The four ROC plots correspond to the cases described as follows. AT1: MDTW applied to the whole signature. AT2: MDTW applied to the whole signature after 3-level wavelet decomposition. AT3: MDTW applied at stroke level. AT4: MDTW applied at stroke level and 3-level wavelet decomposition applied to each stroke. 


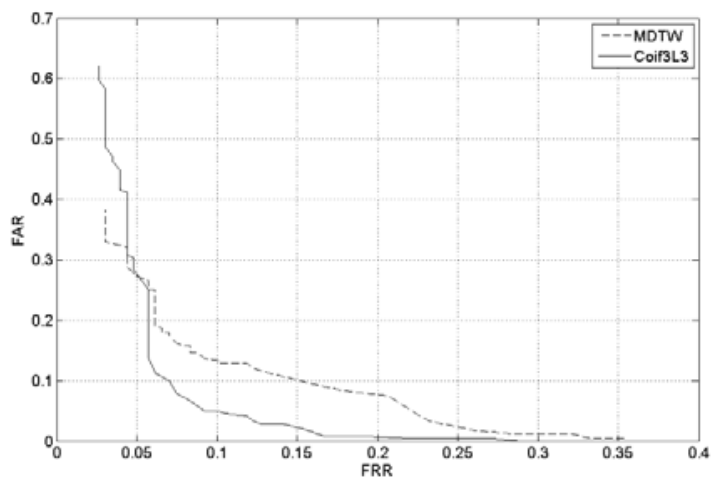

Fig. 8. ROC curves obtained using MDTW direct analysis vs MDTW-wavelet analysis

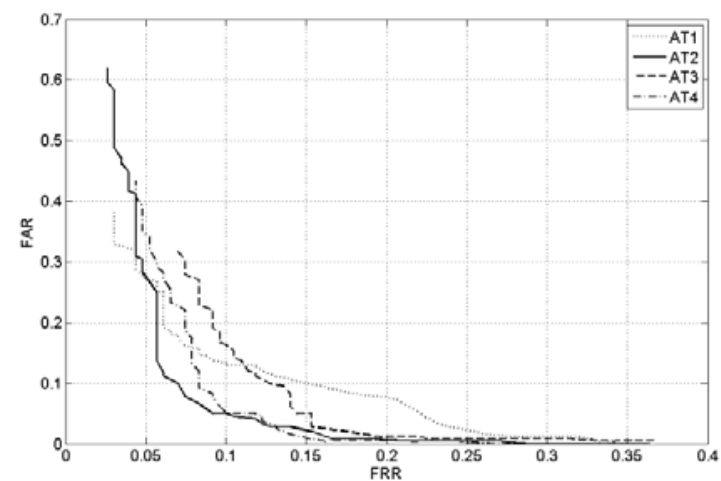

Fig. 9. ROC curves obtained using the MDTW algorithm in four different cases

\section{Conclusions}

This paper presented a signature-based biometric system using a modified dynamic time warping algorithm and wavelet decomposition. The described modification on the DTW when compared to the classical algorithm, provided an improvement in the system performance of $3.07 \%$ in average, as represented in the corresponding ROC curves. A further incorporation of a wavelet-based decomposition gave an additional improvement in the system performance, as well as a dimensionality reduction, which provided a considerable decreasing in execution time with an estimated factor of 23 , when it was compared with the execution time without the wavelet decomposition. The best obtained results using both techniques showed in average a combined $\mathrm{EER}=7.46 \%$. Further experiments using larger databases are currently in progress. 


\section{References}

1. Jain, A.K.: Handbook of Biometrics. Springer, Heidelberg (2008)

2. Bowyer, K.W., Hollingsworth, K., Flynn, P.J.: Image understanding for iris biometrics: A survey. Computer Vision and Image Understanding 110(2), 281-307 (2008)

3. Chellappa, R., Sinha, P., Jonathon Phillips, P.: Face Recognition by Computers and Humans. Computer 43(2), 46-55 (2010)

4. Nanni, L., Lumini, A.: A multi-matcher for ear authentication. Pattern Recognition Letters 28, 2219-2226 (2007)

5. Ramírez-Cortes, J.M., Gómez-Gil, P., Sánchez-Pérez, G., Prieto-Castro, C.: Shape-based hand recognition approach using the pattern spectrum. Journal of Electronic Imaging 18(1) (2009)

6. Maltoni, D., Maio, D., Jain, A.K., Prabhakar, S.: Handbook of fingerprint recognition, 2nd edn. Springer, Heidelberg (2009)

7. Kong, A., Zhang, D., Kamel, M.: A survey of palmprint recognition. Pattern Recognition 42(7), 1408-1418 (2009)

8. Kozik, R., Choras, M.: Combined Shape and Texture Information for Palmprint Biometrics. Journal of Information Assurance and Security 5, 058-063 (2010)

9. Impedovo, D., Pirlo, G.: Automatic signature verification: The state of the art. IEEE Trans. Syst. Man, Cybern. C 38(5) (September 2008)

10. McCabe, A., Trevathan, J., Read, W.: Neural Network-based Handwritten Signature Verification. Journal of Computers 3(8) (August 2008)

11. Fierrez, J., Ortega-Garcia, J., Ramos, D., Gonzalez-Rodriguez, J.: HMM-based on-line signature verification: Feature extraction and signature modeling. Pattern Recognition Letters 28(16), 2325-2334 (2007)

12. Nanni, L., Lumini, A.: A novel local on-line signature verification system. Pattern Recognition Letters 29(5), 559-568 (2008)

13. Khalid, M., Mokayed, H., Yusof, R., Ono, O.: Online Signature Verification with Neural Networks Classifier and Fuzzy Inference. In: 2009 Third Asia International Conference on Modelling \& Simulation, pp. 236-241 (May 2009)

14. Nanni, L., Maiorana, E., Lumini, A., Campisi, P.: Combining local, regional and global matchers for a template protected on-line signature verification system. Expert Systems with Applications 37(5), 3676-3684 (2010)

15. Yanikoglu, B., Kholmatov, A.: Online Signature Verification Using Fourier Descriptors. EURASIP Journal on Advances in Signal Processing (2009) Article ID 260516

16. Priestley, M.B.: Wavelets and time-dependent spectral analysis. Journal of Time Series Analysis 17(1), 85-103 (2008)

17. Pinsky, M.A.: Introduction to Fourier Analysis and Wavelets. Graduate Studies in Mathematics, vol. 102. American Mathematical Society, Providence (2009)

18. Kruskal, J.B., Liberman, M.: The symmetric time-warping problem: from continuous to discrete. In: Sanko, D., Kruskal, J.B. (eds.) Time Warps, String Edits, and Macromolecules: The Theory and Practice of Sequence Comparisons, pp. 125-160. Addison-Wesley, Reading (1983)

19. Dunstone, T., Yager, N.: Biometric System and Data Analysis: Design. Evaluation and Data Mining. Springer, New York (2009) 